\title{
On the Longitude Effect of a Corpuscular Stream from the Sun
}

\author{
By G. ISHIKAWA \\ Meteorological Research Institute
}

Many of the investigations hitherto made on the production of the ionosphere are based on the photo-ionization process and the results obtained seem to be valid for $E$-layer. There remain, however, many obscurities about the features of $F$-layer, of which the most essential concerns the diurnal variation of its electron density. The diurnal variation of the electron density in $F$-layer is so asymmetric that we ought to consider that it arises from an asymmetric distribution of incident ray. Such a possibility is hardly conceived so long as we adopt the photo-ionization process for the production of $F$-layer. But it may be considered likely if we visualize a charged particle in place of a photon as an ionizing source. A corpuscular stream coming in one direction from a place very distant from the earth will be affected by the earth's magnetic field so that the longitudinal distribution of incident particles near the earth should be asymmetric. The shape of such a distribution may be governed both by momentum and impact parameter of corpuscles.

Let us introduce rectangular coordinates $x, y, z$ with origin at the earth's dipole ( $z$-axis coincides with the magnetic axis) and assume that the direction of $x$-axis is in parallel with that of the motion of the stream at the long distance of $L$ from the origin, where the field may be regarded as nearly equal to zero.

So that the initial state of a particle is given by the coordinates $s=(y)_{x=L}$ and $z_{0}=(z)_{x=L}$.

The equation of motion of a particle of mass $m$ carrying a charge $e$ is written as usual :

$$
\dot{\boldsymbol{v}}=\frac{e}{m}(\boldsymbol{v} \times \boldsymbol{H})
$$

where $\boldsymbol{v}$ is the velocity of a particle and $\boldsymbol{H}$ is the magnetic field produced by a dipole of moment $M$. A perfect solution of this equation is not easily found, but numerical treatments are carried out by several authors. [1], [2], [3], [4] What is necessary here is not to find a perfect solution of trajectories, but to consider the general character of the possible solution. Making a transformation of variables from rectangular to polar coordinates $r, \theta, \varphi$ we obtain the solutions of equation (1):

$$
\begin{aligned}
& r=r\left(t, p, s, z_{0}\right) \\
& \theta=\theta\left(t, p, s, z_{0}\right) \\
& \varphi=\varphi\left(t, p, s, z_{0}\right)
\end{aligned}
$$

where $p$ is the momentum of a corpuscle, which is conserved throughout the motion, 
Then the position $(\theta, \varphi)$ of a particle of initial condition $\left(p, s, z_{0}\right)$ at the earth's surface $(r=R)$ is readily obtained from the above solutions:

$$
\begin{aligned}
& \theta=f_{1}\left(p, s, z_{0}\right) \\
& \varphi=f_{2}\left(p, s, z_{0}\right)
\end{aligned}
$$

So that the surface density $\sigma$ of corpuscles at the earth's surface is given by:

$$
\sigma\left(p, s, z_{0}\right) R^{2} \sin \theta d \theta d \varphi=\sigma_{0} d s d z_{0}
$$

where $\sigma_{0}$ is the surface density of the stream of momentum $p$ at $x=L$ plane. Eliminating $s$ and $z_{0}$ from eqs. (3) and (4) we have:

$$
\sigma(\theta, \varphi, p)=\sigma_{0} f(\theta, \varphi, p)
$$

This gives the longitudinal (together with the latitudinal) effect or the diurnal variation of incident intensity of a monochromatic corpuscular stream of momentum $p$.

If the stream has a momentum spectrum $\sigma_{0}(p) d p$ in the initial state, the required density distribution of the incident stream may be written as:

$$
\sigma(\theta, \varphi)=\int_{p_{0}}^{\infty} \sigma_{0}(p) f(\theta, \varphi, p) d p
$$

where the lower limit $p_{0}$ is the critical momentum with which the particle can reach the earth's surface from infinity.

The density distribution of incident particles at the earth's surface is thus governed both by a definite function $f(\theta, \varphi, p)$ and the momentum spectrum $\sigma_{0}(p) d p$.

Hence we may obtain a convenient distribution of $\sigma$ for explaining the asymmetric and double maximal diurnal variation of $F$-layer, if we choose a suitable momentum spectrum in the corpuscular stream. The only points to examine are firstly whether such $\sigma_{0}(p)$ is likely or not and secondly whether the ionization by a corpuscle of such a momentum is enough to explain that actually observed in $F$-layer.

The treatment of the general case is not so easy and here we mention the special case of two dimentional problem in the equitorial plane. In this case we can readily obtain for the equation (3):

(A) for $s>-2 C$

$$
\varphi=\int_{R}^{\infty} \frac{\left(s r+C^{2}\right) d r}{r\left\{r^{4}-\left(s r+C^{2}\right)^{2}\right\}^{\frac{1}{2}}},
$$

where

$$
C=\sqrt{e \bar{M} / p}
$$

is the well-known Störmer unit of length. The equation (7) is expressed in elliptic integral as usuals :

$$
\varphi=\frac{\pi}{2}-\sin ^{-1}(K \sin \psi)+\frac{s}{2 \sqrt{2} C} F(K, \phi),
$$

where

$$
\begin{aligned}
& \dot{K}=\sqrt{4 C^{2}+s^{2}} / 2 \sqrt{2} C, \\
& \phi=\cos ^{-1}\left\{-\frac{s r+2 C^{2}}{r \sqrt{4 C^{2}+s^{2}}}\right\},
\end{aligned}
$$


and $F(K, \psi)$ is the elliptic integral of the first kind.

(B) for $s<-2 C$

$$
\varphi=\frac{\pi}{2}-\psi-\frac{s}{\sqrt{4 C^{2}+s^{2}}} F\left(K^{\prime}, \phi\right),
$$

where

$$
\begin{aligned}
K^{\prime} & =\frac{2 \sqrt{2} C}{\sqrt{4 C^{2}+s^{2}}}, \\
\psi & =\sin ^{-1} \sqrt{\frac{r^{2}-s r-C^{2}}{2 r^{2}}} .
\end{aligned}
$$

For the equation (4) we have:

$$
\sigma=\sigma_{0} / R\left(\frac{\partial \varphi}{\partial s}\right)_{r=R} .
$$

Using the equation (9) to (11), we obtain :

(A)

$$
\begin{aligned}
\frac{\partial \varphi}{\partial s}= & \frac{4 s C^{2} r^{3}-s^{4} r^{2}+8 C^{4} r^{2}-3 s^{3} C^{2} r-2 s^{2} C^{4}}{r\left(16 C^{4}-s^{4}\right) \sqrt{r^{4}-\left(s r+C^{2}\right)^{2}}} \\
& +\frac{\sqrt{2} C}{4 C^{2}+s^{2}} F(K, \phi)+\frac{2 \sqrt{2} C s^{2}}{16 C^{4}-s^{4}} E(K, \psi),
\end{aligned}
$$

where $E(K, \psi)$ is the elliptic integral of the second kind.

(B)

$$
\begin{aligned}
\frac{\partial \varphi}{\partial s}= & \frac{C^{2}\left[\left(s^{4}-16 C^{4}\right) r^{2}+4 s^{2}\left\{r^{4}-\left(s r+C^{2}\right)^{2}\right\}\right]}{r\left(s r+2 C^{2}\right)\left(s^{4}-16 C^{4}\right) \sqrt{r^{4}-\left(s r+C^{2}\right)^{2}}} \\
& -\frac{1}{\sqrt{4 C^{2}+s^{2}}} F\left(K^{\prime}, \phi\right)+\frac{s^{2}}{\sqrt{4 C^{2}+s^{2}}\left(s^{2}-4 C^{2}\right)} E\left(K^{\prime}, \phi\right) .
\end{aligned}
$$

The function $f(\varphi, p)$ is easily obtained using the eqs. (5), (12) and (13). Finally the lower limit $p_{0}$ in the equation (6) is given in this case by:

$$
p_{0}=\frac{e M}{(\sqrt{2}+1)^{2} R^{2}} \text {. }
$$

[1] C. Störmer: Zeits. für Astrophys. 1, 237 (1930), Terr. Mag. 3, 31 (1931).

[2] M.S. Vallarta: Univ. of Toronto Studies, Applied Math. Series. 3 (1938).

[3] M.S. Vallarta, C. Graef and S. Kusaka: Phys. Rev. 55, 1 (1939).

[4] T. Yagi: Cosmic Ray Research, Nagoya Univ. III, 2, 13 (1949). 